\title{
Magnetic Resonance with Squeezed Microwaves
}

\author{
A. Bienfait, ${ }^{1,2}$ P. Campagne-Ibarcq, ${ }^{1}$ A. H. Kiilerich, ${ }^{3}$ X. Zhou, ${ }^{1,4}$ S. Probst, ${ }^{1}$ J. J. Pla, ${ }^{5}$ T. Schenkel, ${ }^{6}$ \\ D. Vion, ${ }^{1}$ D. Esteve, ${ }^{1}$ J. J. L. Morton, ${ }^{7}$ K. Moelmer, ${ }^{3}$ and P. Bertet ${ }^{1}$ \\ ${ }^{1}$ Quantronics group, SPEC, CEA, CNRS, Université Paris-Saclay, \\ CEA Saclay 91191 Gif-sur-Yvette Cedex, France \\ ${ }^{2}$ Institute for Molecular Engineering, University of Chicago, Chicago, Illinois 60637, USA \\ ${ }^{3}$ Department of Physics and Astronomy, Aarhus University, \\ Ny Munkegade 120, DK-8000 Aarhus C, Denmark \\ ${ }^{4}$ Institute of Electronics Microelectronics and Nanotechnology, CNRS UMR 8520, ISEN Department, \\ Avenue Poincaré, CS 60069, 59652 Villeneuve d'Ascq Cedex, France \\ ${ }^{5}$ School of Electrical Engineering and Telecommunications, University of New South Wales, \\ Anzac Parade, Sydney, NSW 2052, Australia \\ ${ }^{6}$ Accelerator Technology and Applied Physics Division, Lawrence Berkeley National Laboratory, \\ Berkeley, California 94720, USA \\ ${ }^{7}$ London Centre for Nanotechnology, University College London, London WC1H OAH, United Kingdom
}

(Received 22 May 2017; published 17 October 2017)

\begin{abstract}
Vacuum fluctuations of the electromagnetic field set a fundamental limit to the sensitivity of a variety of measurements, including magnetic resonance spectroscopy. We report the use of squeezed microwave fields, which are engineered quantum states of light for which fluctuations in one field quadrature are reduced below the vacuum level, to enhance the detection sensitivity of an ensemble of electronic spins at millikelvin temperatures. By shining a squeezed vacuum state on the input port of a microwave resonator containing the spins, we obtain a 1.2-dB noise reduction at the spectrometer output compared to the case of a vacuum input. This result constitutes a proof of principle of the application of quantum metrology to magnetic resonance spectroscopy.
\end{abstract}

DOI: 10.1103/PhysRevX.7.041011

Subject Areas: Magnetism, Quantum Physics,

Quantum Information

\section{INTRODUCTION}

The detection and characterization of electron spins in a sample by magnetic resonance spectroscopy [1] has numerous applications in materials science, chemistry, and quantum information processing. Pulsed magnetic resonance detection proceeds by detecting weak microwave signals emitted by spins resonant with a cavity in which the sample is embedded. The noise present in these signals determines the spectrometer sensitivity and is ultimately limited by the fluctuations in the microwave field at the cavity output. The thermal contribution to these fluctuations can be removed by lowering the temperature $T$ of the sample and cavity such that $k_{B} T \ll \hbar \omega_{s}$, where $\omega_{s}$ is the spin resonance frequency and $k_{B}$ is Boltzmann's constant [2]. However, even at these cryogenic temperatures, quantum fluctuations of the electromagnetic field remain and pose a fundamental limitation to the achievable sensitivity.

Published by the American Physical Society under the terms of the Creative Commons Attribution 4.0 International license. Further distribution of this work must maintain attribution to the author(s) and the published article's title, journal citation, and DOI.
Field fluctuations are governed by Heisenberg's uncertainty principle, which states that $\delta X^{2} \delta Y^{2} \geq 1 / 16$. In this expression, $\hat{X}$ and $\hat{Y}$ are the two quadrature operators of the field in dimensionless units, normalized such that $\left\langle\hat{X}^{2}\right\rangle+\left\langle\hat{Y}^{2}\right\rangle=N+1 / 2, N$ being the average photon number in the field mode of interest. When the field is in a coherent state, which is the case for the echo signals emitted by the spins, $\delta X^{2}=\delta Y^{2}=1 / 4$, as in the vacuum state. It is possible, however, to engineer so-called squeezed states in which the variance in one quadrature (called the squeezed quadrature) is reduced below $1 / 4$, at the expense of an increase in variance in the other quadrature, as required by Heisenberg's inequality (see Fig. 1). Most experiments demonstrating the production $[3,4]$ and use of squeezed states have been performed in the optical domain. Squeezed optical states have been used to enhance the sensitivity of interferometric measurements [5-7] with applications in gravitational wave detection [8,9], atomic absorption spectroscopy [10], imaging [11], atom-based magnetometry [12], and of particle tracking in biological systems [13].

At microwave frequencies, the need to operate at cryogenic temperatures and the lack of applications limited the interest in squeezed states to pioneering proof-ofprinciple demonstrations [14] until the recent advent of 


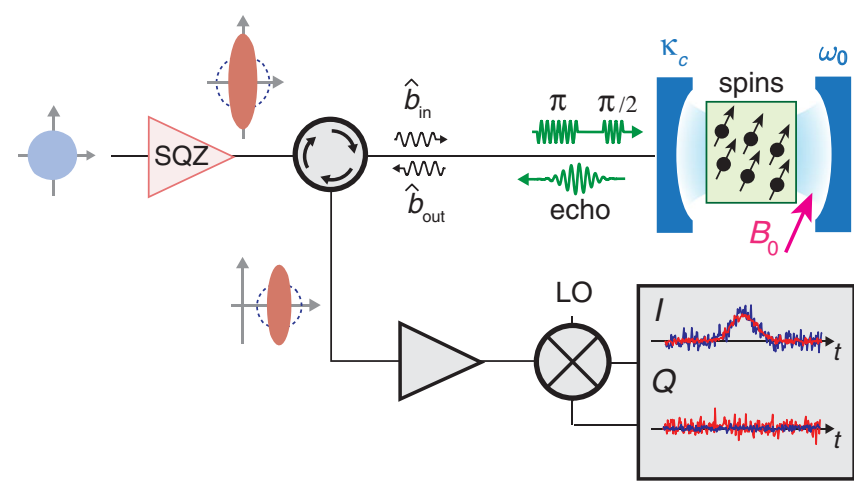

FIG. 1. Principle of squeezing-enhanced pulsed magnetic resonance. A squeezed vacuum state is incident on an ESR cavity of frequency $\omega_{0}$. The cavity contains the spins to be detected, which are tuned into resonance at $\omega_{0}$ by a dc magnetic field $B_{0}$. A Hahn echo microwave pulse sequence $(\pi / 2-\tau-\pi-\tau)$ is applied to the spins, leading to the emission of an echo in the detection waveguide on the $X$ quadrature. This echo is noiselessly amplified along $X$ before its homodyne demodulation with a local oscillator (LO) phase such that $I(t)$ is proportional to $X(t)$. The traces in the bottom right-hand gray box, which are not real data, depict schematically the expected difference between SQZ off (blue) and SQZ on (red) output quadrature signals when the squeezed quadrature is aligned along the echo emission quadrature $X$; the signal-to-noise ratio is improved on the $I$ quadrature, which contains the entire echo signal.

quantum information processing with superconducting circuits [15], which requires the control and measurement of microwave fields at the quantum level. This triggered the development of practical Josephson parametric amplifier (JPA) devices [16-19] and follow-up amplifier chains such that the output noise is dominated by quantum fluctuations [20]. Microwave squeezed states [20] can then provide a sizable noise reduction, thus improving measurement sensitivity for qubit state readout [21-24] and nanomechanical resonator motion detection [25]. They have also been investigated for their effect on the dynamics of quantum systems, such as two-level atoms [26-28] or mechanical oscillators [29]. Here, we propose and demonstrate a novel application of quantum squeezing at microwave frequencies to magnetic resonance spectroscopy for improving the detection sensitivity of a small ensemble of electronic spins.

Our scheme is depicted in Fig. 1. A single-port lossless resonator of frequency $\omega_{0}$ containing the sample is coupled with a rate $\kappa_{C}$ to the measurement line that supports incoming $\left(\hat{b}_{\text {in }}\right)$ and outgoing $\left(\hat{b}_{\text {out }}\right)$ field modes. A dc magnetic field $B_{0}$ is applied to tune the spin frequency into resonance with the cavity. A Hahn echo sequence [30] consisting of a $\pi / 2$ pulse at time $t=0$ followed by a $\pi$ pulse at $t=\tau$ leads to rephasing of the spins at $t=2 \tau$ and, whenever $\omega_{s} \approx \omega_{0}$, to the emission of an echo on one field quadrature (that we take here to be $X$ ) in the output measurement line. This echo is then amplified noiselessly with a quantum-limited amplifier tuned to the $X$ quadrature [2] and detected by coherently mixing it with a local oscillator. Throughout this article we consider that the local oscillator phase is adjusted such that the echo signal at the measurement chain output (i.e., after propagation and further amplification) is entirely on one of the two quadratures that we call $I(t)$, the other being denoted $Q(t)$. The noise accompanying the echo arises from the (amplified) fluctuations in $\hat{b}_{\text {in }}$, the input field reflected by the cavity into the output mode; it reaches the quantum limit if $\hat{b}_{\text {in }}$ is in the vacuum state. If $\hat{b}_{\text {in }}$ is instead prepared in a squeezed vacuum state with its squeezed quadrature coinciding with the $X$ quadrature on which the echo is emitted, the noise in $I(t)$ may be below the quantum limit (see Fig. 1), whereas the noise on the other quadrature (which bears no spin signal) is correspondingly increased.

Such a noise reduction at constant echo amplitude implies that the sensitivity of spin-echo detection, defined as the minimum number of spins that can be detected with unit signal-to-noise ratio in a given measurement time, can be improved beyond the limit imposed by vacuum fluctuations of the microwave field simply by sending a squeezed state onto the cavity input. For a given sample, the same signal-to-noise ratio can thus be reached in a shorter measurement time using squeezed states. This may have practical applications in magnetic resonance, in particular for samples with very low spin concentrations, or even containing only one spin [31]. It also raises an interesting fundamental question about the ultimate limits on the signal-to-noise ratio achievable in spin-echo detection, given that the electromagnetic noise contribution can be fully suppressed by combining quantum squeezing and noiseless amplification.

The purpose of this article is twofold. First, in Sec. II, we present a theoretical analysis of the experiment proposed above. The results confirm that, in the limit where the coupling of the spin ensemble to the resonator is weak, squeezed state injection does lead to reduced noise in the echo signal. Second, we present an experimental implementation of this proposal. In Sec. III, we characterize squeezed vacuum radiation generated by a Josephson parametric amplifier at millikelvin temperatures and we analyze the physical effects that limit the noise reduction to $1.2 \mathrm{~dB}$. In Sec. IV, we demonstrate that applying the squeezed microwave source to the ESR spectrometer increases its sensitivity by the same amount. In Sec. V, we conclude with a discussion of the practical and fundamental interest and limitations of our scheme.

\section{SPIN-ECHO EMISSION IN SQUEEZED VACUUM STATE: THEORY}

Our physical system, illustrated in Fig. 1(b), consists of a cavity mode coupled resonantly to $N_{\text {spins }}$ spins and to input and output microwave fields. As we are interested in both 
the mean amplitude and the quantum fluctuations in the output signal, we describe the whole system quantum mechanically. Inhomogeneous broadening and spatial variations of the spins within the ESR cavity lead to different transition frequencies $\omega_{j}$ and coupling strengths $g_{j}$ of the individual spins to the cavity mode. We assume here that the spins are close to resonance with the cavity, and that their mean frequency $\omega_{s}$ is equal to the cavity frequency $\omega_{0}$. In a frame rotating at $\omega_{0}$, the total Hamiltonian of the spins and the resonator mode is

$$
\hat{H}=\hbar \sum_{j}\left[g_{j}\left(\hat{\sigma}_{-}^{(j)} \hat{a}^{\dagger}+\hat{\sigma}_{+}^{(j)} \hat{a}\right)+\frac{\Delta_{j}}{2} \hat{\sigma}_{z}^{(j)}\right],
$$

where $\Delta_{j}=\omega_{j}-\omega_{0}$ denotes the detuning of the $j$ th spin from the cavity resonance frequency, $\hat{a}$ and $\hat{a}^{\dagger}$ denote field annihilation and creation operators, and $\hat{\sigma}_{z}^{(j)}, \hat{\sigma}_{-(+)}^{(j)}$ are Pauli operators describing the spin degrees of freedom.

The quantum-optical input-output formalism [32] yields the following Heisenberg equation for the cavity field operator:

$\dot{\hat{a}}=-i \sum_{j} g_{j} \hat{\sigma}_{-}^{(j)}-\frac{\kappa}{2} \hat{a}+\sqrt{\kappa_{L}} \hat{b}_{\text {loss }}(t)+\sqrt{\kappa_{C}} \hat{b}_{\text {in }}(t)$,

where $\kappa=\kappa_{C}+\kappa_{L}$ is the total cavity damping rate with contributions $\kappa_{C}$ due to the outcoupling and $\kappa_{L}$ due to internal cavity losses. The last two terms in Eq. (2) describe inputs from bath modes: $\hat{b}_{\text {loss }}(t)$ associated with the internal cavity losses and $\hat{b}_{\text {in }}(t)$ associated with the quantized radiation field incident on the cavity.

Rather than solving the complete excitation dynamics of the spins, we assume that ideal $\pi / 2$ and $\pi$ control pulses have been applied to the spins at times $t=-\tau$ and $t=0$, respectively, preparing a state where the spin-excited states have acquired phases $\exp \left(i \Delta_{j} \tau\right)$ with respect to the spin ground states in a frame rotating at $\omega_{s}$. As the spins precess at different frequencies $\Delta_{j}$, they come back in phase at the later time $t=\tau$, and we analyze their coupling to the quantized field during the rephasing of the spins that leads to the emission of an echo of duration $T_{E}$, set by the spin spectral linewidth and the duration of the $\pi / 2$ and $\pi$ control pulses.

To this end, we apply the so-called Holstein-Primakoff approximation [33], which assumes oscillatorlike commutator relations $\left[\hat{\sigma}_{-}^{(j)}, \hat{\sigma}_{+}^{(k)}\right]=\delta_{j k}$ for the spin-lowering operators $\hat{\sigma}_{-}^{(j)}$, and we treat each spin as an oscillator prepared in a coherent state of complex amplitude $\alpha \exp \left(i \Delta_{j} \tau\right)$ at $t=0$. The precession about the spin $z$ axis due to the inhomogeneous distribution of spin excitation energies is equivalent to the rotation of the complex oscillator amplitude, while the oscillator approximation assigns a constant damping rate to the collective transverse spin components and a linear coupling of the spin and field oscillator amplitudes rather than the nonlinear, excitation-dependent one. Since the decay of the transverse spin components is very limited during the time scale of our protocol, describing it with a constant effective rate constant and assuming a linear oscillatorlike coupling to the field is a good approximation. We solve the coupled dynamics of the field mode and the spin ensemble, and we hence need the Heisenberg equation of motion for the spin-lowering operator, which incorporates the coherent state initial condition as a deltafunction excitation pulse at $t=0$ :

$\dot{\hat{\sigma}}_{j}=-\left(\gamma+i \Delta_{j}\right) \hat{\sigma}_{-}^{(j)}-i g_{j} \hat{a}+\alpha e^{i \Delta_{j} \tau} \delta(t)+\sqrt{2 \gamma} \hat{F}_{j}(t)$.

The relaxation rate $\gamma$ represents spin decoherence and is accompanied by quantum Langevin noise sources $\hat{F}_{j}(t)$ with nonvanishing commutators $\left[\hat{F}_{i}(t), \hat{F}_{j}^{\dagger}\left(t^{\prime}\right)\right]=\delta\left(t-t^{\prime}\right) \delta_{i j}$.

An analysis of the beam-splitter-like coupling of the incident, resonator, and outgoing fields [32] yields the input-output relation:

$$
\hat{b}_{\text {out }}(t)=\sqrt{\kappa_{C}} \hat{a}(t)-\hat{b}_{\text {in }}(t) .
$$

Applying Fourier transforms and solving the resulting algebraic set of equations for the coupled spin and field operators leads to the compact and general form of the output field operator,

$$
\begin{aligned}
\tilde{b}_{\text {out }}(\omega)= & -\frac{i q(\omega)}{\sqrt{2 \pi}}+t(\omega) \tilde{f}_{\text {spin }}(\omega)+l(\omega) \tilde{b}_{\text {loss }}(\omega) \\
& +r(\omega) \tilde{b}_{\text {in }}(\omega)
\end{aligned}
$$

where

$$
\begin{aligned}
q(\omega) & =\frac{2 \sqrt{\kappa_{C}} A(\omega)}{\kappa[1+C(\omega)]-2 i \omega}, \\
t(\omega) & =\frac{2 \sqrt{\kappa_{C} \kappa \operatorname{Re}[C(\omega)]}}{\kappa[1+C(\omega)]-2 i \omega}, \\
l(\omega) & =\frac{2 \sqrt{\kappa_{L} \kappa \operatorname{Re}[C(\omega)]}}{\kappa[1+C(\omega)]-2 i \omega},
\end{aligned}
$$

and

$$
r(\omega)=\frac{\kappa_{C}-\kappa_{L}-\left(\kappa_{C}+\kappa_{L}\right) C(\omega)+2 i \omega}{\kappa[1+C(\omega)]-2 i \omega},
$$

are frequency-dependent complex coefficients describing, respectively, the mean field emitted by the spins and Langevin noise operator terms associated with the spins, the resonator internal loss, and reflection of the microwave field on the cavity. 
In Eqs. (5)-(8), the distribution of spin detunings and coupling strengths are incorporated in the frequencydependent ensemble cooperativity,

$$
C(\omega)=\sum_{j} \frac{2 g_{j}^{2}}{\kappa\left(\gamma+i \Delta_{j}-i \omega\right)},
$$

and the amplitude factor,

$$
A(\omega)=\sum_{j} \frac{g_{j} \alpha e^{i \Delta_{j} \tau}}{\gamma+i \Delta_{j}-i \omega} .
$$

The noise operators $\tilde{f}_{\text {spin }}(\omega)=\tilde{F}_{\text {spin }}(\omega) / \sqrt{\kappa \operatorname{Re}[C(\omega)]}$, $\tilde{b}_{\text {loss }}(\omega)$, and $\tilde{b}_{\text {in }}(\omega)$ obey standard commutator relations, e.g., $\left[\tilde{f}_{\text {spin }}(\omega), \tilde{f}_{\text {spin }}^{\dagger}\left(\omega^{\prime}\right)\right]=\delta\left(\omega-\omega^{\prime}\right)$, and the condition $|r(\omega)|^{2}+|t(\omega)|^{2}+|l(\omega)|^{2}=1$ ensures the same commutator relation applies to the output field operators $\tilde{b}_{\text {out }}(\omega)$. We refer to the Supplemental Material for details of the derivation of the general expressions and for analytical results in the special case of a Lorentzian detuning distribution uncorrelated with the coupling strengths [34].

We now turn to the definition of the modes on which the echo is emitted in order to define and estimate the measurement sensitivity. For the sake of simplicity, we assume that the bandwidth $T_{E}^{-1}$ of the spin-echo signal is narrower than the bandwidth of the squeezed radiation and of the resonator. The output signal mode is defined as $\hat{b}_{\text {mode }}=\left(1 / \sqrt{T_{E}}\right) \int_{\tau-T_{E} / 2}^{\tau+T_{E} / 2} \hat{b}_{\text {out }}\left(t^{\prime}\right) d t^{\prime}$, its $\hat{X}$ quadrature operator being $\hat{X}=[1 /(2 i)]\left(\hat{b}_{\text {mode }}-\hat{b}_{\text {mode }}^{\dagger}\right)$. The normalization is chosen such that $\hat{b}_{\text {mode }}^{\dagger} \hat{b}_{\text {mode }}$ is the photon number (operator) in the mode. We similarly introduce $\hat{b}_{\text {in }}=\left(1 / \sqrt{T_{E}}\right) \int_{\tau-T_{E} / 2}^{\tau+T_{E} / 2} \hat{b}_{\text {in }}\left(t^{\prime}\right) d t^{\prime}, \hat{f}_{\text {spin }}=\left(1 / \sqrt{T_{E}}\right) \int_{\tau-T_{E} / 2}^{\tau+T_{E} / 2}$ $\hat{f}_{\text {spin }}\left(t^{\prime}\right) d t^{\prime}$, and $\hat{b}_{\text {loss }}=\left(1 / \sqrt{T_{E}}\right) \int_{\tau-T_{E} / 2}^{\tau+T_{E} / 2} \hat{b}_{\text {loss }}\left(t^{\prime}\right) d t^{\prime}$, as well as their respective $\hat{X}_{\text {in }}, \hat{X}_{\text {bath }}, \hat{X}_{\text {loss }}$ quadrature operators. The mean integrated amplitude of the spin-echo signal is given by the mean value of the $\hat{X}$ operator:

$$
\langle\hat{X}\rangle=\frac{-i q(0)}{\sqrt{T_{E}}} .
$$

From Eq. (5), one obtains that its fluctuations are

$$
\delta X^{2}=|r(0)|^{2} \delta X_{\text {in }}^{2}+|l(0)|^{2} \delta X_{\text {bath }}^{2}+|t(0)|^{2} \delta X_{\text {spin }}^{2} .
$$

In a model where the spins are described as harmonic oscillators coupled via the $F_{j}$ to an effective zero temperature bath, $\delta X_{\text {spin }}^{2}=\frac{1}{4}\left[\left\langle\left[\tilde{f}_{\text {spin }}, \tilde{f}_{\text {spin }}^{\dagger}\right]\right\rangle+2\left\langle\tilde{f}_{\text {spin }}^{\dagger} \tilde{f}_{\text {spin }}\right\rangle\right]$ is equal to $1 / 4$. A more realistic description of the spins, going beyond the Holstein-Primakoff approximation and taking into account a nonzero effective temperature, would yield a larger value but still of order unity. Since
$|t(0)|^{2}=\left[\left(\kappa_{C}\right) / \kappa\right]\left(1-|r(0)|^{2}\right) \simeq 4\left[\left(\kappa_{C}\right) / \kappa\right] C(0)$, the contribution of the spin fluctuation to the total output noise scales as the ensemble cooperativity $C(0)$.

In the limit where the ensemble cooperativity and the cavity losses are small $\left[C(0) \ll 1\right.$ and $\left.|l(0)|^{2} \ll 1\right]$, which is the case in our experiment, as we explain in the following sections, the dominant contribution to the output fluctuations therefore is the reflected input noise and Eq. (13) reduces to $\delta X \approx \delta X_{\text {in }}$. This input field originates from a squeezing source [SQZ in Fig. 1(b)] that we assume to be ideal, generating a squeezed vacuum along the $X$ quadrature with a variance $\delta X_{\mathrm{sq}}^{2}$ at its output. Because of transmission losses between SQZ and the ESR cavity, modeled by an effective loss coefficient $\eta_{\text {loss }}$, the squeezing properties are deteriorated and the variance in the input quadrature becomes

$$
\delta X_{\text {in }}^{2}=\left(1-\eta_{\text {loss }}\right) \delta X_{\mathrm{sq}}^{2}+\eta_{\text {loss }} / 4,
$$

characterized by the ratio of the squeezed quadrature variance to the vacuum fluctuations $\eta_{S}=\delta X_{\text {in }}^{2} /(1 / 4)$ called the squeezing factor. The signal-to-noise ratio of the spinecho detection is given by $\langle\hat{X}\rangle / \delta X,\langle\hat{X}\rangle$ being independent of the input field fluctuations as seen from Eq. (12). Our analysis therefore shows that in the limit where the spin ensemble is weakly coupled to the cavity and the cavity losses are negligible, applying a squeezed vacuum to the ESR resonator should improve the spin-detection sensitivity by approximately a factor $\sqrt{\eta_{S}}$.

\section{EXPERIMENTAL RESULTS: SQUEEZED STATE CHARACTERIZATION}

We now turn to the experimental implementation of this proposal, starting with the description and the characterization of the squeezed microwave source. Squeezing and noiseless amplification are achieved by the same type of device: a flux-pumped JPA operated in the degenerate mode, denoted SQZ for the squeezer and AMP for the amplifier. The JPA consists of a single-port resonator of frequency close to $\omega_{0}$ containing a SQUID array [see Fig. 2(a)]. The magnetic flux threading each SQUID loop is modulated by a pump tone at frequency $2 \omega_{0}$ with a phase $\phi$ leading to a resonator frequency modulation $\propto$ $\cos \left[2\left(\omega_{0} t+\phi\right)\right]$ [18]. Parametric amplification with amplitude gain $G$ occurs for input signals $V \cos \omega_{0} t$ if $\phi=-\pi / 4$, and deamplification with gain $1 / G$ if $\phi=+\pi / 4$. The SQZ is a JPA acting on the vacuum at its input, generating a squeezed vacuum state with a variance reaching $1 /\left(4 G^{2}\right)$ on its squeezed quadrature and an average photon number $N=\left(G^{2}+G^{-2}-2\right) / 4$ (see Supplemental Material [34]).

As we explain in Sec. II, squeezing is very sensitive to microwave losses. It is thus important to characterize the squeezed state with a setup that contains all elements used for the magnetic resonance experiment (described in 


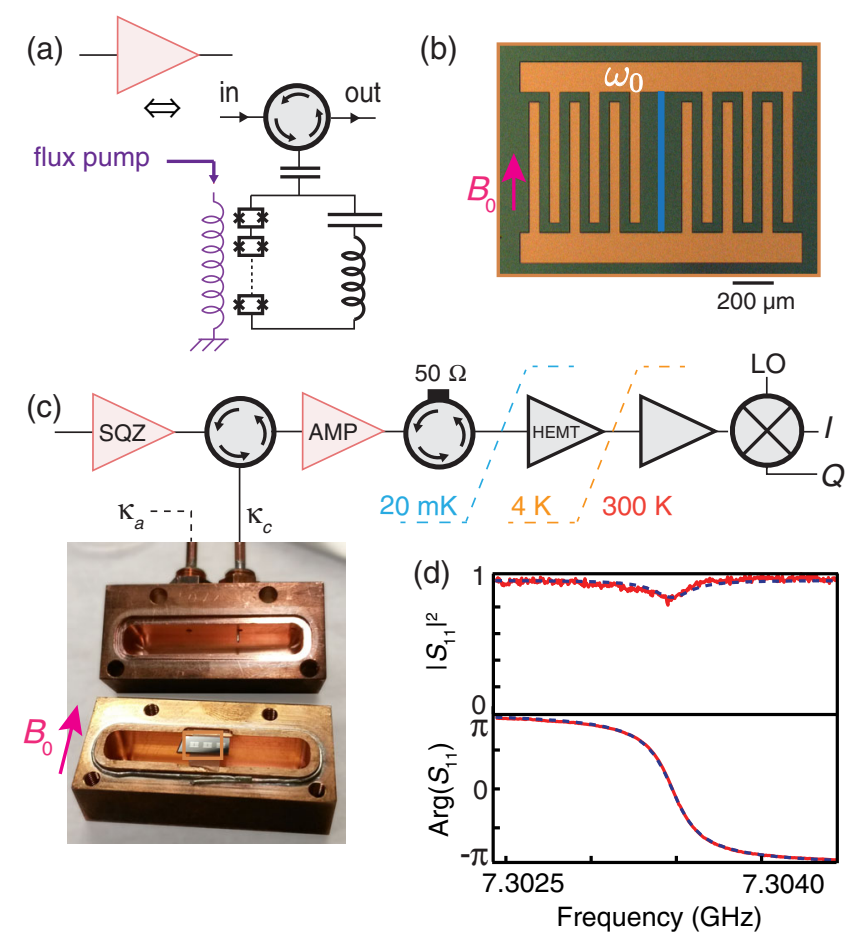

FIG. 2. Experimental setup. (a) The JPAs providing the squeezed vacuum and the noiseless amplification are superconducting $L C$ resonators containing a SQUID array, tuned to a frequency close to the ESR cavity frequency $\omega_{0}$ by the application of a dc flux bias to the SQUID loops. Modulating this flux at twice the resonator frequency by application of a pump tone yields parametric gain at $\omega \simeq \omega_{0}$ for the signals reflected off the JPA. (b) The ESR cavity, whose optical micrograph is shown, is an aluminum lumpedelement $L C$ resonator of frequency $\omega_{0}$ patterned on top of a silicon sample containing the spins. A magnetic field $B_{0}$ is applied parallel to the sample surface and to the resonator inductor (in blue) to tune the spin frequency. Only spins in the immediate vicinity of the inductor are detected. (c) The resonator is enclosed in a copper box holder and is capacitively coupled to the measurement line with a constant $\kappa_{C}$ via an antenna fed through the 3D copper sample holder, thermally anchored at $20 \mathrm{mK}$. A second port, much less coupled (constant $\kappa_{A} \ll \kappa_{C}$ ), is used for characterization (see text). Squeezed microwaves at $\omega_{0}$ are generated by a first JPA denoted $\mathrm{SQZ}$, routed onto the resonator via a circulator, and the reflected signal is noiselessly amplified by a second JPA denoted AMP. Both are operated in the degenerate mode and pumped at $2 \omega_{0}$ with respective phases $\phi_{S}$ and $\phi_{A}$. Further amplification is provided at $4 \mathrm{~K}$ by a high electron mobility transistor (HEMT) amplifier and at $300 \mathrm{~K}$. Homodyne demodulation at the signal frequency yields the quadratures $I(t)$ and $Q(t)$. (d) Measured reflection coefficient $\left|S_{11}^{2}\right|$ (red line; blue line is a fit) yielding $\omega_{0} / 2 \pi=7.3035 \mathrm{GHz}$, $\kappa_{C}=1.6 \times 10^{6} \mathrm{~s}^{-1}$, and $\kappa_{A}+\kappa_{L}=6 \times 10^{4} \mathrm{~s}^{-1}, \kappa_{L}$ being the resonator internal loss rate. We determine $\kappa_{A}=3 \times 10^{3} \mathrm{~s}^{-1}$ by measuring the full resonator scattering matrix (not shown).

Sec. IV), including the ESR cavity. This ESR cavity consists of a high-quality-factor superconducting $L C$ resonator patterned on top of a silicon sample. It is enclosed in a copper box holder and connected to the measurement line by capacitive coupling to an antenna [see Fig. 2(c)], whose length sets the coupling constant $\kappa_{C}$. The squeezed vacuum generated by SQZ is sent into the ESR cavity input via a circulator which routes the reflected field into AMP. Further amplification stages include a semiconductor high-electronmobility transistor (HEMT) amplifier at $4 \mathrm{~K}$ as well as room-temperature amplifiers. Transmission and reflection coefficients can be measured with a network analyzer. For phase-sensitive measurements, a microwave signal at the cavity frequency $\omega_{0}$ is sent into the JPA. After amplification, the output signal is demodulated by mixing with a local oscillator also at $\omega_{0}$, yielding time traces of the quadratures $I(t)$ and $Q(t)$ that are digitized with a $300-\mathrm{kHz}$ bandwidth. More details on the setup can be found in the Supplemental Material [34] and in Ref. [2].

To characterize the squeezed microwave state, we keep the spins detuned from the ESR resonator by working at $B_{0}=0 \mathrm{mT}$. We show in Fig. 3(a) the effect of pump phase $\left(\phi_{S}\right.$ and $\left.\phi_{A}\right)$ on the power gain $\left(G_{S}^{2}\right.$ and $\left.G_{A}^{2}\right)$ for SQZ and AMP, respectively. The gains vary sinusoidally as expected, with $G_{S}^{2}=6 \mathrm{~dB}$ and $G_{A}^{2}=18 \mathrm{~dB}$ for the chosen pump amplitude settings. In the remainder of this work, the local oscillator phase is set such that the quadrature maximally amplified by AMP is $I(t)$. Note that the detection bandwidth is much smaller than the 3-MHz bandwidth of both JPAs. The variance $\delta I^{2}$ is shown in Fig. 3(b) as a function of the relative phase between the SQZ and AMP pump signals $\phi_{\Delta}=\phi_{S}-\phi_{A}$, with no signal at the input. As demonstrated in Ref. [20], $\delta I^{2}$ depends on $\phi_{\Delta}$, allowing us to experimentally determine the optimal squeezing condition $\phi_{\Delta}=\pi / 2$. Statistical distributions of $I(t)$ are shown in the form of histograms in Fig. 3(c) for this optimal condition. We find that the variance in the total output noise $\left(\delta I_{\mathrm{on}}^{2}\right)$ is reduced $1.2 \mathrm{~dB}$ below that observed with the SQZ pump off $\left(\delta I_{\text {off }}^{2}\right), \delta I_{\text {on }}^{2}=0.75 \delta I_{\text {off }}^{2}$ [see Figs. 3(b) and 3(c)].

In order to determine whether this reduced noise is indeed below the vacuum fluctuations level, it is necessary to determine how close $\delta I_{\text {off }}^{2}$ is to the vacuum fluctuations value $\delta I_{0}^{2}$. It is indeed well known that the temperature of the cavity field may differ from the sample temperature of $10 \mathrm{mK}$, due to leakage of thermal radiation from highertemperature stages. Calibration measurements (reported in the Supplemental Material [34]) are performed using a transmon qubit and enable us to put an upper bound of $\bar{n}=0.1$ thermal photon present in the mode. Since in a thermal state the variance of a quadrature $\hat{X}$ is given by $\left\langle\delta X^{2}\right\rangle=(1+2 \bar{n}) / 4$, we can state that in our experiment $\delta I_{0}^{2}<\delta I_{\text {off }}^{2}<1.2 \delta I_{0}^{2}$. We thus come to the conclusion that the measured noise with SQZ on is lower than the vacuum fluctuations by at least $10 \%$ and at best by $25 \%$, proving that the produced squeezed state is truly in the quantum regime.

The measured noise reduction is limited by several factors, one of them being the sensitivity of squeezed 
(a)

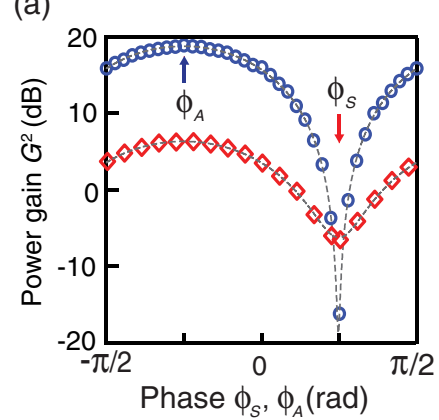

(b)

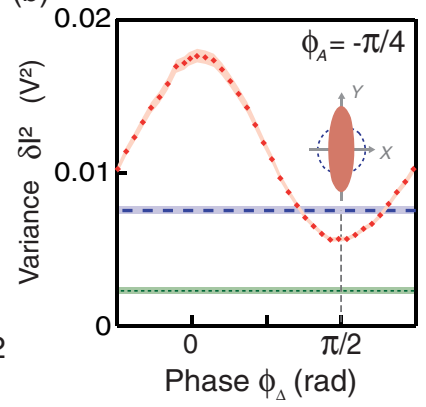

(c)

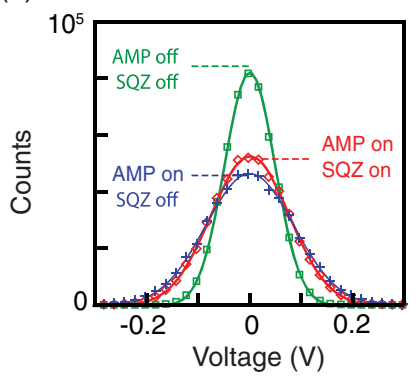

(d)

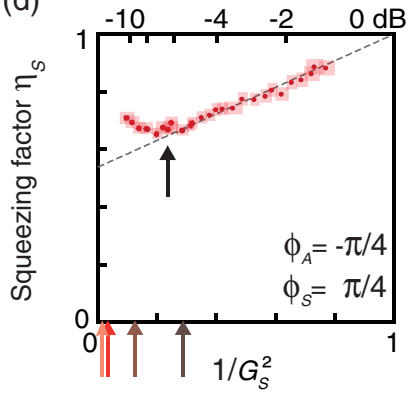

FIG. 3. Characterization of the prepared squeezed vacuum state. (a) Gain of SQZ (open red diamonds) and AMP (open blue circles) as a function of their pump phase ( $\phi_{S, A}$, respectively) for the chosen pump amplitude settings, leading to a power gain $G_{S}^{2}=6 \mathrm{~dB}$ and $G_{A}^{2}=20 \mathrm{~dB}$ for $\phi_{S, A}=-\pi / 4$. Optimal values for the SQZ and AMP pump phases are indicated with arrows. Dashed curves are sinusoidal fits. (b) The variance $\delta I^{2}$ in the noise is plotted as a function of the difference between the SQZ and AMP pump phases $\phi_{\Delta}=\phi_{S}-\phi_{A}, \phi_{A}$ being set at its optimal value. Data with AMP and SQZ both on (red diamonds) are compared to those obtained with AMP on and SQZ off (blue dashed line), and with AMP and SQZ both off (green dashed line). Shaded areas represent the $5 \sigma$ measurement uncertainty. Squeezing is obtained for the optimal setting $\phi_{\Delta}=\pi / 2$. (c) Noise histograms obtained using the optimal phases obtained above, for AMP and SQZ both off (green open squares), in which case the noise is determined by the HEMT amplifier, AMP on and SQZ off (blue crosses), in which case the noise is the sum of the HEMT and the amplified vacuum fluctuations, and AMP and SQZ both on (red open symbols), in which case the fluctuations are reduced below the vacuum level. Gaussian fits for each are also shown (curves). (d) Using the optimal phase settings, the squeezing factor (see main text) $\eta_{S}$ is measured as a function of the SQZ power gain (red dots; rectangles represent the $5 \sigma$ measurement uncertainty) by varying the SQZ pump power. A linear fit (dashed line) for the low-gain part of the curve indicates the microwave losses between SQZ and AMP to be $\eta_{\text {loss }}=0.54$. The black arrow indicates the SQZ gain selected in the experiment.

states to losses, as we discuss in Sec. II. For our experiment, the relevant microwave losses are those between SQZ and AMP, which include the insertion loss of circulators and cables and internal losses of the SQZ and AMP devices as well as of the ESR resonator. Care is taken to minimize these losses; in particular, the coupling rate of the resonator to the output waveguide, $\kappa_{C}=1.2 \times 10^{6} \mathrm{~s}^{-1}$, is purposely

set to be much larger than the internal loss rate of the resonator, $\kappa_{L}=6 \times 10^{4} \mathrm{~s}^{-1}$, so that the losses in reflection are below $1 \mathrm{~dB}$, as shown in Fig. 2(d).

To quantify these losses, we measure the squeezing factor $\eta_{S} \equiv\left(\delta I_{\mathrm{on}}^{2}-\delta I_{\mathrm{bg}}^{2}\right) /\left(\delta I_{\mathrm{off}}^{2}-\delta I_{\mathrm{bg}}^{2}\right)$ as a function of $G_{S}^{2}$ [see Fig. 3(d)], $\delta I_{\text {bg }}^{2}$ being the variance in the background noise obtained when both SQZ and AMP are switched off. With this definition, $\eta_{S}$ measures only the quantum noise reduction due to squeezing; according to Eq. (14), one expects $\eta_{S}=\eta_{\text {loss }}+\left(1-\eta_{\text {loss }}\right) / G_{S}^{2}$. At low gain $(\lesssim 5 \mathrm{~dB})$, $\eta_{S}$ is indeed measured to depend linearly on $G_{S}^{-2}$. A linear fit yields $\eta_{\text {loss }}=0.54$.

At higher gain, a departure from linearity is observed, with an increase of the variance. To investigate this phenomenon, we measure the SQZ response to a coherent input signal having an amplitude corresponding to the rootmean-square vacuum fluctuations, with the SQZ operated in degenerate mode. Varying this signal phase $\phi$ from 0 to $2 \pi$, the output quadratures $\left[X_{\text {out }}(\phi), Y_{\text {out }}(\phi)\right]$ mimic the shape of the produced squeezed vacuum, as seen in Fig. 4(a). At small or moderate gains $\left(G_{S}^{2}<6 \mathrm{~dB}\right)$, an ellipse is observed with its small-axis projection scaling as $G_{S}^{-2}$. For gains larger than $10 \mathrm{~dB}$, however, the ellipse becomes strongly distorted, which explains the increase of the squeezed quadrature variance at high gain observed in Fig. 3(d). We attribute this ellipse distortion phenomenon to cubic or quartic nonlinearities in the parametric amplifier Hamiltonian, arising from higher-order terms in the expansion of the Josephson junction potential energy [36,37]. We therefore choose to set the SQZ pump power such that $G_{S}^{2}=6 \mathrm{~dB}$, which yields the largest amount of squeezing, as seen in Fig. 3(d).

The nonlinearity of the JPA devices also affects the operation of the noiseless amplifier AMP, by causing power-dependent phase shifts and saturation of the output power, as seen in Fig. 4(b). Power-dependent phase shifts are particularly detrimental for our experiment: squeezed states have a higher power than the vacuum, which implies that the echo signal may be phase shifted when the squeezing is turned on, leading to a reduced output amplitude. We illustrate this effect by sending a small coherent pulse onto the ESR resonator via an additional port [see Fig. 2(c)] whose coupling rate to the ESR resonator $\kappa_{A} \ll \kappa_{L}, \kappa_{C}$ is negligible. The phase of the coherent pulse and the SQZ pump are set so that the pulse is detected on the $I$ quadrature and is aligned with the squeezed vacuum. Figure 4(c) shows the recorded time traces $I(t)$ with squeezing switched on and off for different input powers. While for powers below $-136 \mathrm{dBm}$ the amplitudes observed with squeezing on and off are identical as desired, at larger input powers there is a difference by a few percent. To avoid this effect in the experiment described in the next section, we limit the power of the spin-echo signal well below this threshold. 

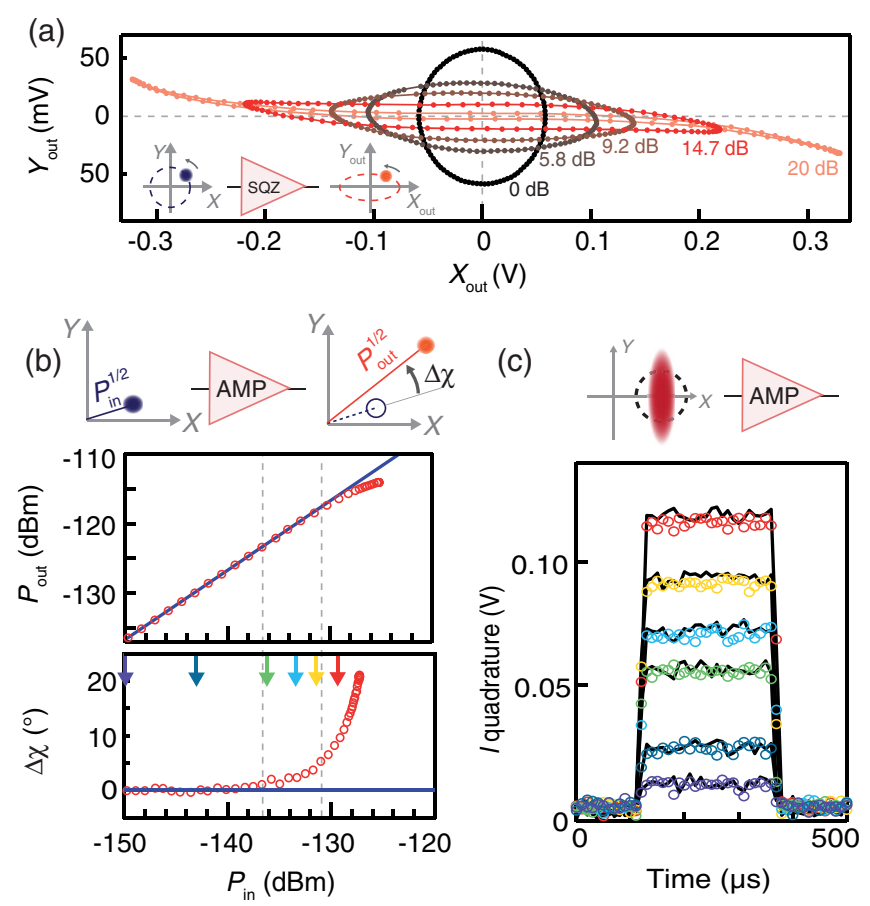

FIG. 4. Limitations induced by JPA saturation. (a) Output quadratures $X_{\text {out }}$ and $Y_{\text {out }}$ measured for weak coherent signals sent to the SQZ with input phases $\phi_{S}$ spanning the whole interval between 0 and $2 \pi$ for 4 different gains $G_{S}^{-2}$ indicated by the arrows in Fig. 3(d) and set using different pump powers (black curve corresponds to SQZ pump off). Note that these data are obtained in a separate calibration run in which the ESR cavity is removed [35]. (b) Measured output power and phase of a signal at $\omega_{0}$ as a function of its input power $P_{\text {in }}$ after amplification by the AMP device, in the same conditions as in (a) but with the JPA operated in the nondegenerate mode by detuning the pump by $300 \mathrm{kHz}$ from $2 \omega_{0}$. The output power depends linearly on $P_{\text {in }}$ as long as $P_{\text {in }}<-131 \mathrm{dBm}$ (blue lines is a linear fit), while the phase shift is zero (blue line) only for $P_{\text {in }}<-137 \mathrm{dBm}$. (c) Time trace of the $I$ quadrature of a weak microwave pulse at $\omega_{0}$ sent via the $\kappa_{A}$ port of the ESR cavity, measured with SQZ off (open circles) and SQZ on (black traces) for different input powers indicated by the arrows in (b). The traces have been averaged 4000 times. Above $-136 \mathrm{dBm}$, deviations appear between the SQZ on and SQZ off curves due to AMP saturation.

\section{ESR SPECTROSCOPY IN THE PRESENCE OF SQUEEZING}

The spins we use in the experiment are provided by bismuth $\left({ }^{209} \mathrm{Bi}\right)$ donors implanted in the silicon sample, which has been isotopically enriched in nuclear-spin-free ${ }^{28} \mathrm{Si}$. At low magnetic fields, the strong hyperfine interaction between the $S=1 / 2$ electron and the $I=9 / 2$ nuclear spins yields multiple allowed ESR-like transitions around $7.37 \mathrm{GHz}$ [see Fig. 5(a)]; we work here on the lowest frequency transition. More details on the characterization of this sample can be found in Refs. [38,39].

Figure 5(b) shows this spin resonance line, obtained by measuring the spin-echo intensity as a function of the

(a)
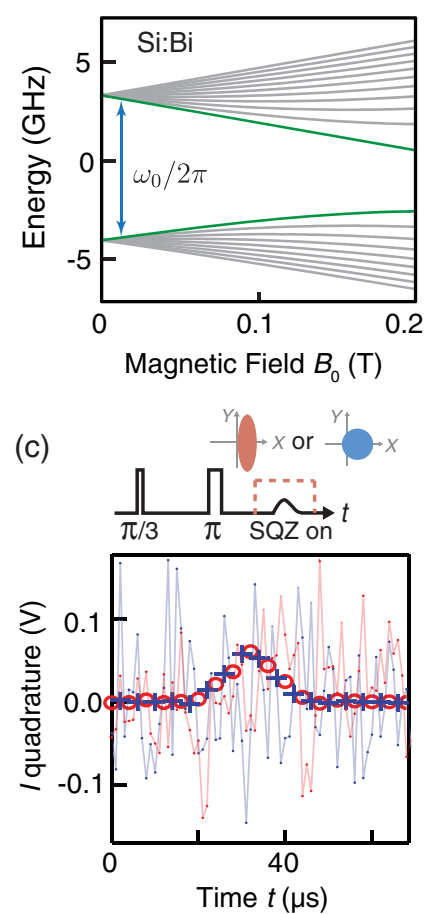

(b)

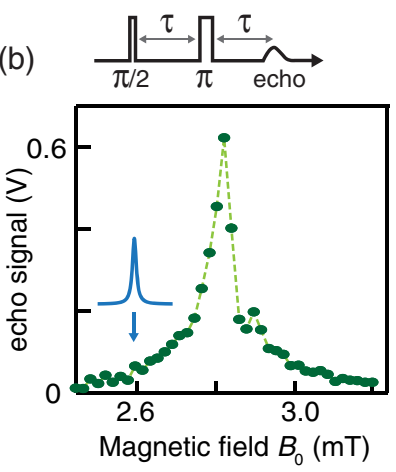

(d)

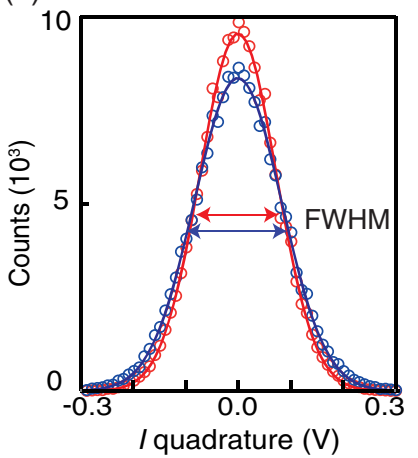

FIG. 5. Squeezing-enhanced spin-echo detection. (a) Energy of the 20 levels of bismuth donors in silicon as a function of $B_{0}$ (gray lines). The transition between the levels indicated in green is used in the experiment. (b) Hahn-echo-detected magnetic field sweep, showing the bismuth donor resonance line. Blue arrow indicates the field chosen in the rest of the experiment, blue Lorentzian curve indicates the fraction of spins that are within the cavity resonance. (c) Echo signals observed with SQZ off (blue) and on (red) for a single shot (lines) and averaged over 2500 traces (symbols) confirm the signal intensity is identical. SQZ was switched on only during a short $\Delta t=200-\mu$ s window around the echo emission time (dashed rectangle in the pulse sequence). The excitation pulse angle is chosen to be $\approx \pi / 3$ in order to avoid saturation effects (see main text). (d) Histograms of the noise around the average signals of (c) measured with 2500 single-shot traces acquired on a 70- $\mu$ s time window centered on the echo, with SQZ off (blue) and on (red) (see Supplemental Material [34]), and corresponding Gaussian fits (curves). Standard deviations are $0.0858 \pm 2 \times 10^{-4} \mathrm{~V}$ for SQZ off and $0.0748 \pm 2 \times$ $10^{-4} \mathrm{~V}$ for SQZ on, confirming a reduction in the noise accompanying the spin-echo signal when SQZ is on. 
Rabi oscillations (obtained by sweeping the power of the 10- $\mu$ s rectangular refocusing pulse in the Hahn echo sequence) are used to calibrate the pulses for subsequent experiments [41]. To avoid saturation of AMP as explained in the previous section, we purposely set the field far away from the maximum of the spin resonance line at $B_{0}=$ $2.6 \mathrm{mT}$ [see Fig. 5(b)] and we use an echo sequence of the form $\theta-\tau-\pi-\tau-$ echo in which the usual $\pi / 2$ Rabi angle of the Hahn echo is replaced by a lower Rabi angle $\theta$ (the $\theta \simeq \pi / 3$ rotation is realized via a $3-\mu$ s rectangular pulse) with $\tau=200 \mu \mathrm{s}$.

An echo is then recorded in the two following conditions: SQZ off and SQZ pump switched on for a time window of $200 \mu$ s centered around the echo emission time (pulsing the squeezed state generation is found to be crucial for the success of the experiment, for reasons we explain in the next section). The phases of the excitation and refocusing pulses are set in such a way that the echo signal is produced entirely on the $I$ quadrature aligned with the squeezed vacuum. Time traces of the digitized $I(t)$ quadrature are shown in Fig. 5(c), with the echo barely visible in single-shot traces. After averaging, the spin-echo amplitude appears to be identical for SQZ on and off, confirming that the saturation effects mentioned in Sec. III are avoided. The $20-\mu$ s echo duration arises from the excitation bandwidth. Histograms of the noise during the echo emission [Fig. 5(d)] show that the data obtained with SQZ on exhibit less noise than with SQZ off-indeed, the similarity between these distributions and those obtained with no spin-echo signal [Fig. 3(c)] confirm that quantum fluctuations are the primary noise source in the spin-echo measurements. In both cases the variance is reduced by a factor of 0.75 when SQZ is on, in agreement with the theoretical analysis presented in Sec. II. As the noise reduction is obtained while maintaining constant spin-echo signal amplitude, this demonstrates that the sensitivity of magnetic resonance detection is enhanced using quantum squeezing.

\section{DISCUSSION AND CONCLUSION}

\section{A. Applicability of the scheme}

We now discuss to what extent our proof-of-principle demonstration of squeezing-enhanced magnetic resonance detection can be improved to be of practical use. The measured noise reduction of $1.2 \mathrm{~dB}$ provided by the squeezed state injection is limited by the finite value of the input field squeezing factor $\eta_{S}$, as seen in Fig. 3(d). As explained in Sec. III, this is due in our experiment to two distinct phenomena. The first one is the nonlinearity of both the SQZ and the AMP parametric amplifiers, which puts a lower bound on $\eta_{S}$ and limits the maximum spin signal that can be amplified and detected. All these issues can be solved by using other JPAs with up to 30-dB higher saturation power than our design $[42,43]$. Keeping everything else unchanged in our experiment, we estimate that using these optimized amplifiers would have resulted in a noise reduction of $\approx 3 \mathrm{~dB}$. The second factor limiting the squeezing-induced noise reduction is the presence of microwave losses on the path over which the squeezed state propagates; those are due to cables, circulators, and resonator internal losses. As explained in Sec. III, they add up to $\approx 3 \mathrm{~dB}$ in our setup, but simple improvements (minimizing cable length, using a lower number of circulators, and a resonator of lower internal losses) could bring this figure down to $1 \mathrm{~dB}$. These straightforward improvements realistically lead to a noise reduction on the squeezed state quadrature by a factor of 5 below the vacuum level. The resulting reduction of the measurement time by a factor of 5 with unchanged signal-to-noise ratio is clearly relevant for practical applications. To reduce the losses below $1 \mathrm{~dB}$, more radical setup changes would probably be needed, such as integrating the squeezer, the circulator, the ESR resonator, and the amplifier on a single chip. While such a complex quantum integrated circuit has never been achieved so far, promising steps in that direction have already been taken, with, in particular, several recent demonstrations of on-chip superconducting circulators [44-47].

When considering the practical relevance of our scheme and results for magnetic resonance spectroscopy, one point deserves attention. Because microwave losses must be minimized to preserve the degree of squeezing of the field, the resonator should be largely overcoupled to the measurement line (i.e., $\kappa_{C} \gg \kappa_{L}$ ). However, the measurement sensitivity of a spin-echo detection scales like $\kappa^{-1 / 2}$ [2], $\kappa=\kappa_{C}+\kappa_{L}$ being the total resonator loss rate. Increasing $\kappa_{C}$ thus reduces the measurement sensitivity in the absence of squeezing. In our experiment, for instance, we estimate that $N_{\text {min }}=1.3 \times 10^{4}$ spins can be detected with a signalto-noise ratio of 1 per echo without squeezing (see Supplemental Material [34]), a factor of 7 larger than what was achieved with a critically coupled resonator [2]. One can thus wonder whether it is really more desirable for ESR measurements to use an overcoupled resonator with squeezed microwaves sent onto its input, instead of a critically coupled resonator without any squeezing. Focusing exclusively on spin sensitivity, however, neglects the fact that most electron spin species in general have a linewidth that is much broader than the $\approx 10-50-\mathrm{kHz}$ linewidth of a critically coupled superconducting resonator [2]. In this case, the lower intrinsic spin sensitivity of an overcoupled resonator is compensated by the larger number of spins measured, resulting in unchanged signal-to-noise ratio for spin-echo measurements. In other words, it is desirable to match the resonator linewidth to the spin linewidth; with superconducting resonators this amounts to overcoupling the resonator to the measurement line, exactly as required for the squeezing enhancement. Note that lowering the resonator quality factor comes with other 
advantages, such as the possibility of applying largebandwidth control pulses. To sum up, squeezing-enhanced ESR spectroscopy is well suited to measure spins whose linewidth is comparable or larger than the one of an overcoupled superconducting resonator $(\approx 1 \mathrm{MHz}$, i.e., $0.03 \mathrm{mT}$ ), which is the case in our experiment as well as for many spin species [1].

\section{B. Ultimate limits to the sensitivity and squeezing backaction on the spin dynamics}

After discussing the applicability of our scheme, we now wish to address a more fundamental question. Supposing that an ideal squeezed state of arbitrary squeezing factor could be sent onto the cavity so that the purely electromagnetic contribution to the total noise would be completely suppressed, it is then worthwhile to investigate which other physical mechanisms would ultimately limit the sensitivity. Those can be deduced from Eq. (13), which shows that in the limit where $\delta X_{\text {in }} \rightarrow 0$, a finite variance is maintained, $\delta X^{2}=|l(0)|^{2} \delta X_{\text {bath }}^{2}+|t(0)|^{2} \delta X_{\text {spin }}^{2}$. In an ideal experiment, the cavity internal losses may be suppressed so that the first term is negligible; however, the second term describes noise emitted by the spins (as observed experimentally in Ref. [48]), and is thus unavoidable. Negligible in our experiment, this contribution becomes relevant in the limit where the squeezing factor $\eta_{S}$ becomes comparable to the ensemble cooperativity $4 C(0)$, as seen in Sec. II. Additional measurements (reported in the Supplemental Material [34]) yield $C(0)=0.002$, which implies that spin noise would be a limitation for 20 -dB squeezing, corresponding to a maximum gain in sensitivity by a factor $\simeq 10$.

Another fundamental effect disregarded so far concerns the effect of squeezed radiation on the spin dynamics, which may in certain cases lead to a reduction of the echo signal. Indeed, in steady state, squeezed radiation incident on a two-level system modifies its relaxation and coherence times as well as its average polarization, as predicted in Ref. [26] and observed in recent experiments with superconducting qubits $[28,49]$. Note that squeezing does not affect the damping rates of a harmonic oscillator, which explains its absence in the analysis of Sec. II, where the spins are modeled as a collection of oscillators [50].

To investigate experimentally these effects, we measure spin coherence and relaxation times with SQZ turned off or on during the entire experimental sequence. $T_{2}$ is found to be unaffected by squeezing [see Fig. 6(a)], because decoherence occurs by nonradiative processes such as dipolar interactions [51]. Energy relaxation, on the other hand, has been shown to be caused by spontaneous emission of microwave photons through the cavity (the Purcell effect) with a rate $T_{1}^{-1}=4 g^{2} / \kappa$ [41], $g$ being the coupling of a single spin to the radiation field as defined in Sec. II. Being of radiative origin, the effective $T_{1}$ should be modified by the squeezed microwave injection. Accordingly, it is found to decrease from 0.9 to $0.45 \mathrm{~s}$ when (a)
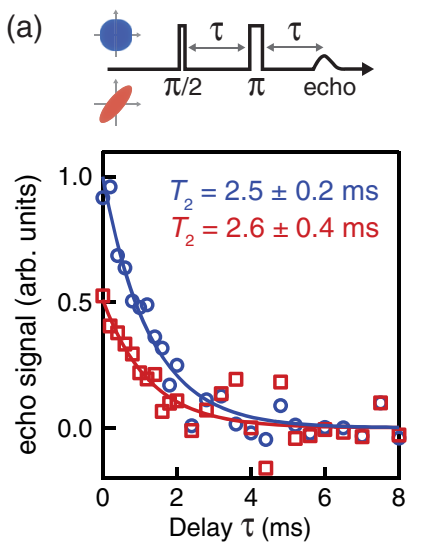

(b)
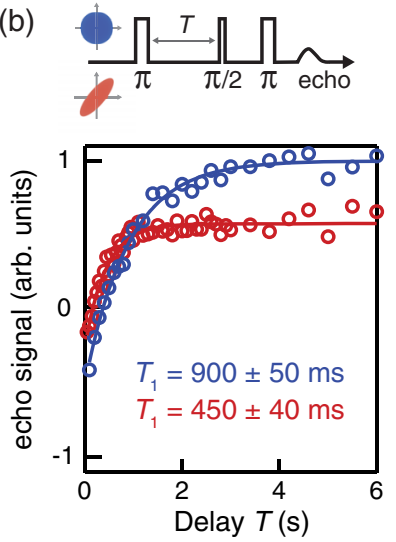

FIG. 6. Influence of squeezing on the spin coherence times. (a) Coherence time $T_{2}$ measured with a Hahn echo sequence for SQZ off (blue circles) and on (red squares). Contrary to the experiments in Fig. 5, SQZ is now switched on or off for the entire experimental sequence. The integrated echo signal is plotted as a function of the delay $\tau$ between the $\pi / 2$ and $\pi$ pulses. Exponential fits (solid lines) yield $T_{2, \text { off }}=2.5 \pm 0.2 \mathrm{~ms}$ and $T_{2, \text { on }}=2.6 \pm 0.4 \mathrm{~ms}$. (b) Energy relaxation time $T_{1}$ with SQZ on (blue circles) and off (red circles). Exponential fits (solid lines) yield $T_{1}=900 \pm 50 \mathrm{~ms}$ with SQZ off and $T_{1}=450 \pm$ $40 \mathrm{~ms}$ with SQZ off. Both $T_{1}$ and $T_{2}$ curves [(a) and (b)] have their amplitude reduced by $\approx 0.5$ with SQZ on, indicating reduced spin polarization in the steady state when the SQZ is continuously switched on.

squeezing is continuously switched on [see Fig. 6(b)], with an overall echo amplitude diminished by the same factor 2 , revealing the expected reduced spin polarization. The reduction factor on both $T_{1}$ and polarization is predicted by Gardiner to be $1+2 N$ [26], yielding $N \approx 0.5$ in our experiment, compatible with the chosen SQZ gain $G_{S}^{2}=6 \mathrm{~dB}$ as well as with the squeezed state characterization by homodyne detection shown in Fig. 3 .

Squeezing-induced spin depolarization is avoided in the data shown in Fig. 3(c) because SQZ is switched on only for a short time window $\Delta t=200 \mu \mathrm{s}$ around the echo, much smaller than the depolarization time which is of order $\approx T_{1} /(1+2 N)=0.45 \mathrm{~s}$ in our experiment. This strategy can be applied only if the depolarization time is longer than the echo duration $T_{E}$, i.e., if the squeezing parameter $\eta_{S} \approx 1 / N>8 g^{2} T_{E} / \kappa$. It is interesting to note that $8 g^{2} T_{E} / \kappa \approx 4 C(0) / N_{\text {spins }}$, which is the single-spin cooperativity and is therefore much smaller than the ensemble cooperativity $4 C(0)$ as long as the ensemble contains a large number of spins $N_{\text {spins }} \gg 1$. Spin noise is therefore expected to limit the achievable sensitivity gain much earlier than spin depolarization, provided the squeezed state generation is pulsed as in the present experiment. For our experimental parameters, $T_{1} / T_{E} \approx 10^{-5}$, so that squeezing-induced spin depolarization would not be an issue unless $50-\mathrm{dB}$ squeezing is reached, instead of the 20-dB limit found for spin noise. 


\section{Conclusion}

In conclusion, we present a proof-of-principle demonstration of squeezing-enhanced magnetic resonance detection. While the fundamental limitations to this scheme deserve further study, our results could likely be improved using present-day technologies to gain up to a factor of 5 in measurement time, reaching the point at which the method becomes practically relevant for magnetic resonance measurements. Besides improving the degree of squeezing, future work could investigate the use of other nonclassical states, such as Schrödinger-cat states in magnetic resonance, which might bring even larger sensitivity gains $[52,53]$.

\section{ACKNOWLEDGMENTS}

We acknowledge technical support from P. Sénat, D. Duet, J.-C. Tack, P. Pari, and P. Forget, as well as useful discussions within the Quantronics group. We acknowledge the support of the European Research Council under the European Community's Seventh Framework Programme (FP7/2007-2013) through Grant Agreements No. 615767 (CIRQUSS), No. 279781 (ASCENT), and No. 630070 (quRAM), and of the Agence Nationale de la Recherche under the project QIPSE. J. J. L. M. is supported by the Royal Society. T. S. was supported by the U.S. Department of Energy under Contract No. DE-AC0205CH11231. A. H. K. and K. M. acknowledge support from the Villum Foundation.

[1] A. Schweiger and G. Jeschke, Principles of Pulse Electron Paramagnetic Resonance (Oxford University Press, New York, 2001).

[2] A Bienfait, J. J. Pla, Y. Kubo, M. Stern, X. Zhou, C.-C. Lo, C. D. Weis, T. Schenkel, M. L. W. Thewalt, D. Vion, D. Esteve, B. Julsgaard, K. Moelmer, J. J. L. Morton, and P. Bertet, Reaching the Quantum Limit of Sensitivity in Electron Spin Resonance, Nat. Nanotechnol. 11, 253 (2015).

[3] R. E. Slusher, L. W. Hollberg, B. Yurke, J. C. Mertz, and J. F. Valley, Observation of Squeezed States Generated by Four-Wave Mixing in an Optical Cavity, Phys. Rev. Lett. 55, 2409 (1985).

[4] L.-A. Wu, H. J. Kimble, J. L. Hall, and H. Wu, Generation of Squeezed States by Parametric Down Conversion, Phys. Rev. Lett. 57, 2520 (1986).

[5] C. M. Caves, Quantum-Mechanical Noise in an Interferometer, Phys. Rev. D 23, 1693 (1981).

[6] P. Grangier, R. E. Slusher, B. Yurke, and A. LaPorta, Squeezed-Light-Enhanced Polarization Interferometer, Phys. Rev. Lett. 59, 2153 (1987).

[7] M. Xiao, L.-A. Wu, and H. J. Kimble, Precision Measurement beyond the Shot-Noise Limit, Phys. Rev. Lett. 59, 278 (1987).
[8] J. Abadie et al. (LIGO Scientific Collaboration), A Gravitational Wave Observatory Operating beyond the Quantum Shot-Noise Limit, Nat. Phys. 7, 962 (2011).

[9] J. Aasi, J. Abadie, B. P. Abbott, R. Abbott, T. D. Abbott, M. R. Abernathy, C. Adams, T. Adams, P. Addesso, R. X. Adhikari et al., Enhanced Sensitivity of the LIGO Gravitational Wave Detector by Using Squeezed States of Light, Nat. Photonics 7, 613 (2013).

[10] E. S. Polzik, J. Carri, and H. J. Kimble, Spectroscopy with Squeezed Light, Phys. Rev. Lett. 68, 3020 (1992).

[11] N. Treps, N. Grosse, W. P. Bowen, C. Fabre, H.-A. Bachor, and P. K. Lam, A Quantum Laser Pointer, Science 301, 940 (2003).

[12] V. G. Lucivero, R. Jiménez-Martínez, J. Kong, and M. W. Mitchell, Squeezed-Light Spin Noise Spectroscopy, Phys. Rev. A 93, 053802 (2016).

[13] M. A. Taylor, J. Janousek, V. Daria, J. Knittel, B. Hage, H.-A. Bachor, and W. P. Bowen, Biological Measurement beyond the Quantum Limit, Nat. Photonics 7, 229 (2013).

[14] R. Movshovich, B. Yurke, P. G. Kaminsky, A. D. Smith, A. H. Silver, R. W. Simon, and M. V. Schneider, Observation of Zero-Point Noise Squeezing via a JosephsonParametric Amplifier, Phys. Rev. Lett. 65, 1419 (1990).

[15] M.H. Devoret and R. J. Schoelkopf, Superconducting Circuits for Quantum Information: An Outlook, Science 339, 1169 (2013).

[16] M. A. Castellanos-Beltran and K. W. Lehnert, Widely Tunable Parametric Amplifier Based on a Superconducting Quantum Interference Device Array Resonator, Appl. Phys. Lett. 91, 083509 (2007).

[17] N. Bergeal, F. Schackert, M. Metcalfe, R. Vijay, V.E. Manucharyan, L. Frunzio, D. E. Prober, R. J. Schoelkopf, S. M. Girvin, and M. H. Devoret, Phase-Preserving Amplification Near the Quantum Limit with a Josephson Ring Modulator, Nature (London) 465, 64 (2010).

[18] X. Zhou, V. Schmitt, P. Bertet, D. Vion, W. Wustmann, V. Shumeiko, and D. Esteve, High-Gain Weakly Nonlinear Flux-Modulated Josephson Parametric Amplifier Using a SQUID Array, Phys. Rev. B 89, 214517 (2014).

[19] C. Macklin, K. OBrien, D. Hover, M. E. Schwartz, V. Bolkhovsky, X. Zhang, W. D. Oliver, and I. Siddiqi, A Near-Quantum-Limited Josephson Traveling-Wave Parametric Amplifier, Science 350, 307 (2015).

[20] F. Mallet, M. A. Castellanos-Beltran, H. S. Ku, S. Glancy, E. Knill, K. D. Irwin, G. C. Hilton, L. R. Vale, and K. W. Lehnert, Quantum State Tomography of an Itinerant Squeezed Microwave Field, Phys. Rev. Lett. 106, 220502 (2011).

[21] N. Didier, A. Kamal, W. D. Oliver, A. Blais, and A. A. Clerk, Heisenberg-Limited Qubit Read-Out with TwoMode Squeezed Light, Phys. Rev. Lett. 115, 093604 (2015).

[22] N. Didier, J. Bourassa, and A. Blais, Fast Quantum Nondemolition Readout by Parametric Modulation of Longitudinal Qubit-Oscillator Interaction, Phys. Rev. Lett. 115, 203601 (2015).

[23] A. Eddins, S. Schreppler, D. M. Toyli, L. S. Martin, S. Hacohen-Gourgy, L. C. G. Govia, H. Ribeiro, A. A. Clerk, and I. Siddiqi, Stroboscopic Qubit Measurement with Squeezed Illumination, arXiv:1708.01674. 
[24] L. C. G. Govia and A. A. Clerk, Enhanced Qubit Readout Using Locally Generated Squeezing and Inbuilt PurcellDecay Suppression, New J. Phys. 19, 023044 (2017).

[25] J. B. Clark, F. Lecocq, R. W. Simmonds, J. Aumentado, and J. D. Teufel, Observation of Strong Radiation Pressure Forces from Squeezed Light on a Mechanical Oscillator, Nat. Phys. 12, 683 (2016).

[26] C. W. Gardiner, Inhibition of Atomic Phase Decays by Squeezed Light: A Direct Effect of Squeezing, Phys. Rev. Lett. 56, 1917 (1986).

[27] K. W. Murch, S. J. Weber, K. M. Beck, Eran Ginossar, and I. Siddiqi, Reduction of the Radiative Decay of Atomic Coherence in Squeezed Vacuum, Nature (London) 499, 62 (2013).

[28] D. M. Toyli, A. W. Eddins, S. Boutin, S. Puri, D. Hover, V. Bolkhovsky, W. D. Oliver, A. Blais, and I. Siddiqi, Resonance Fluorescence from an Artificial Atom in Squeezed Vacuum, Phys. Rev. X 6, 031004 (2016).

[29] J. B. Clark, F. Lecocq, R. W. Simmonds, J. Aumentado, and J. D. Teufel, Sideband Cooling beyond the Quantum Backaction Limit with Squeezed Light, Nature (London) 541, 191 (2017).

[30] E. L. Hahn, Spin Echoes, Phys. Rev. 80, 580 (1950).

[31] P. Haikka, Y. Kubo, A. Bienfait, P. Bertet, and K. Mølmer, Proposal for Detecting a Single Electron Spin in a Microwave Resonator, Phys. Rev. A 95, 022306 (2017).

[32] C. W. Gardiner and M. J. Collett, Input and Output in Damped Quantum Systems: Quantum Stochastic Differential Equations and the Master Equation, Phys. Rev. A 31, 3761 (1985).

[33] T. Holstein and H. Primakoff, Field Dependence of the Intrinsic Domain Magnetization of a Ferromagnet, Phys. Rev. 58, 1098 (1940).

[34] See Supplemental Material at http://link.aps.org/ supplemental/10.1103/PhysRevX.7.041011 for additional details on the calculations, experimental setup, and measurement protocol.

[35] A. Bienfait, Ph.D. thesis, Université Paris-Saclay, 2016.

[36] W. Wustmann and V. Shumeiko, Parametric Resonance in Tunable Superconducting Cavities, Phys. Rev. B 87, 184501 (2013).

[37] S. Boutin, D. M. Toyli, A. V. Venkatramani, A. W. Eddins, I. Siddiqi, and A. Blais, Effect of Higher-Order Nonlinearities on Amplification and Squeezing in Josephson Parametric Amplifiers, arXiv:1708.00020.

[38] G. Wolfowicz, A. M. Tyryshkin, R. E. George, H. Riemann, N. V. Abrosimov, P. Becker, H.-J. Pohl, M. L. W. Thewalt, S. A. Lyon, and J. J. L. Morton, Atomic Clock Transitions in Silicon-Based Spin Qubits, Nat. Nanotechnol. 8, 561 (2013).

[39] C. D. Weis, C. C. Lo, V. Lang, A. M. Tyryshkin, R. E. George, K. M. Yu, J. Bokor, S. A. Lyon, J. J. L. Morton, and T. Schenkel, Electrical Activation and Electron Spin Resonance Measurements of Implanted Bismuth in Isotopically Enriched Silicon-28, Appl. Phys. Lett. 100, 172104 (2012).

[40] J. J. Pla, A. Bienfait, G. Pica, J. Mansir, F. A. Mohiyaddin, A. Morello, T. Schenkel, B. W. Lovett, J. J. L. Morton, and
P. Bertet, Strain-induced spin resonance splittings in silicon devices, arXiv:1608.07346.

[41] A. Bienfait, J. J. Pla, Y. Kubo, X. Zhou, M. Stern, C.-C. Lo, C. D. Weis, T. Schenkel, D Vion, D. Esteve, J. J. L. Morton, and P. Bertet, Controlling Spin Relaxation with a Cavity, Nature (London) 531, 74 (2016).

[42] J. Y. Mutus, T. C. White, R. Barends, Yu Chen, Z. Chen, B. Chiaro, A. Dunsworth, E. Jeffrey, J. Kelly, A. Megrant, C. Neill, P. J. J. O’Malley, P. Roushan, D. Sank, A. Vainsencher, J. Wenner, K. M. Sundqvist, A. N. Cleland, and John M. Martinis, Strong Environmental Coupling in a Josephson Parametric Amplifier, Appl. Phys. Lett., 104, 263513 (2014).

[43] T. Roy, S. Kundu, M. Chand, A. M. Vadiraj, A. Ranadive, N. Nehra, M. P. Patankar, J. Aumentado, A. A. Clerk, and R. Vijay, Broadband Parametric Amplification with Impedance Engineering: Beyond the Gain-Bandwidth Product, Appl. Phys. Lett. 107, 262601 (2015).

[44] J. Kerckhoff, K. Lalumière, B. J. Chapman, A. Blais, and K. W. Lehnert, On-Chip Superconducting Microwave Circulator from Synthetic Rotation, Phys. Rev. Applied 4, 034002 (2015).

[45] K. M. Sliwa, M. Hatridge, A. Narla, S. Shankar, L. Frunzio, R. J. Schoelkopf, and M. H. Devoret, Reconfigurable Josephson Circulator/Directional Amplifier, Phys. Rev. X 5, 041020 (2015).

[46] B. J. Chapman, E. I. Rosenthal, J. Kerckhoff, B. A. Moores, L. R. Vale, G. C. Hilton, K. Lalumière, A. Blais, and K.W. Lehnert, Widely Tunable On-Chip Microwave Circulator for Superconducting Quantum Circuits, arXiv: 1707.04565 .

[47] F. Lecocq, L. Ranzani, G. A. Peterson, K. Cicak, R. W. Simmonds, J. D. Teufel, and J. Aumentado, Nonreciprocal Microwave Signal Processing with a Field-Programmable Josephson Amplifier, Phys. Rev. Applied 7, 024028 (2017).

[48] T. Sleator, E. L. Hahn, C. Hilbert, and J. Clarke, NuclearSpin Noise, Phys. Rev. Lett. 55, 1742 (1985).

[49] K. W. Murch, S. J. Weber, C. Macklin, and I. Siddiqi, Observing Single Quantum Trajectories of a Superconducting Quantum Bit, Nature (London) 502, 211 (2013).

[50] A. H. Kiilerich and K. Mølmer, Relaxation of an Ensemble of Two-Level Emitters in a Squeezed Bath, arXiv: 1708.00659.

[51] A. M. Tyryshkin, S. Tojo, J. L. Morton, H. Riemann, N. V. Abrosimov, P. Becker, H.-J. Pohl, T. Schenkel, M. L. W. Thewalt, K. M. Itoh, and S. A. Lyon, Electron Spin Coherence Exceeding Seconds in High-Purity Silicon, Nat. Mater. 11, 143 (2012).

[52] V. Giovannetti, S. Lloyd, and L. Maccone, QuantumEnhanced Measurements: Beating the Standard Quantum Limit, Science 306, 1330 (2004).

[53] A. Facon, E.-K. Dietsche, D. Grosso, S. Haroche, J.-M. Raimond, M. Brune, and S. Gleyzes, A Sensitive Electrometer Based on a Rydberg Atom in a Schrödinger-Cat State, Nature (London) 535, 262 (2016). 